\title{
Representation functions, Sidon sets and bases
}

\author{
by \\ G. Grekos (St-Etienne), L. Haddad (Paris), \\ C. Helou (Media, PA) and J. Pinko (Helsinki)
}

1. Introduction. Given a subset $A$ of $\mathbb{N}=\{0,1,2, \ldots\}$ and a positive integer $h$, the $h$-representation function by $A$ is the function which to each integer $n \geq 0$ associates the number $r_{h}(A, n)$ of $h$-tuples of elements of $A$ whose sum is equal to $n$. The study of such functions, their properties and their characterizations are the focus of much attention in additive number theory. In particular, they are used to study some important notions such as that of basis. The set $A$ is called an $h$-basis (resp. an asymptotic $h$-basis) of $\mathbb{N}$ if every integer $n \geq 0$ (resp. every sufficiently large integer $n$ ) is the sum of $h$ elements of $A$. The set $A$ is called a Sidon set if all the sums $a+b$, with $a, b \in A$ and $a \leq b$, are distinct, i.e. if $r_{2}(A, n) \leq 2$ for all integers $n \geq 0$. An open problem due to P. Erdős, A. Sárközy and V. T. Sós [1] asks if there exists a Sidon set which is an asymptotic 3-basis of $\mathbb{N}$. This problem was mistakenly presented in [3] as asking if a Sidon set can be an asymptotic 2-basis of $\mathbb{N}$. Even though the negative answer to the latter question is an easy consequence of some well-known properties, it is not extant in published explicit form, and it would therefore not be without interest to give a proof using some new ideas.

In this paper, we give some new properties of the 2-representation function of an infinite subset $A$ of $\mathbb{N}$, of intrinsic interest. We then apply them to give a simple proof of the fact that a Sidon set cannot be an asymptotic 2-basis of $\mathbb{N}$. We then turn to the real open problem of the existence of a Sidon set which is an asymptotic 3 -basis of $\mathbb{N}$, and we provide a partial answer by proving that a Sidon set cannot be a 3 -basis of $\mathbb{N}$. We also give an algorithm for finding, if any exists, a Sidon set $A$ such that, from a specific point on, every integer is the sum of three elements of $A$.

2000 Mathematics Subject Classification: 11B13, 11B34, 11B75.

Key words and phrases: 2-representation function, Sidon set, asymptotic 2-basis, asymptotic 3-basis. 
2. Definitions and notations. Let $A=\left\{a_{1}<a_{2}<\cdots\right\}$ be a (finite or infinite) subset of the set $\mathbb{N}=\{0,1,2, \ldots\}$ of natural numbers. Let $h$ be a positive integer. The $h$-representation function $r_{h}(A, \cdot)$ by $A$ is defined on $\mathbb{N}$ by

$$
r_{h}(A, n)=\left|\left\{\left(x_{1}, \ldots, x_{h}\right) \in A^{h}: x_{1}+\cdots+x_{h}=n\right\}\right|,
$$

where $n \in \mathbb{N}$, and $|E|$ denotes the cardinality of a set $E$.

The set $A$ is called a Sidon set if $r_{2}(A, n) \leq 2$ for all $n \in \mathbb{N}$. This property is equivalent to the assertion that all the sums $a+b$, for $a, b \in A$ with $a \leq b$, are distinct, which in turn is equivalent to asserting that all the non-zero differences $a-b$, for $a, b \in A$, are distinct.

The set $A$ is called an $h$-basis, or a basis of order $h$, of $\mathbb{N}$ if $r_{h}(A, n)>0$ for all $n \in \mathbb{N}$. It is called an asymptotic $h$-basis, or an asymptotic basis of order $h$, of $\mathbb{N}$ if $r_{h}(A, n)>0$ for all large enough integers $n$. More specifically, given $c \in \mathbb{N}$, we say that $A$ is a $c$-asymptotic $h$-basis of $\mathbb{N}$ if $r_{h}(A, n)>0$ for all $n \geq c$.

The set of all sums of $h$ terms from $A$ is written $h A=\left\{x_{1}+\cdots+x_{h}\right.$ : $\left.\left(x_{1}, \ldots, x_{h}\right) \in A^{h}\right\}$ or $A+\cdots+A$ ( $h$ summands). On the other hand, the set of $h$-multiples of the elements of $A$ is written $h \cdot A=\{h a: a \in A\}$. Clearly, $A$ is an $h$-basis (resp. a $c$-asymptotic $h$-basis) of $\mathbb{N}$ if and only if $h A=\mathbb{N}$ (resp. the interval of integers $[c, \infty[$ is contained in $h A$ ).

We further set $s_{h}(A)=\sup \left\{r_{h}(A, n): n \in \mathbb{N}\right\}$ in $\mathbb{N} \cup\{\infty\}$. Thus $A$ is a Sidon set if and only if $s_{2}(A) \leq 2$.

An old conjecture of Erdös and Turán [2] states that $s_{2}(A)$ is infinite for every asymptotic 2-basis $A$. In other words, if $s_{2}(A)$ is finite, then $r_{2}(A, \cdot)$ takes the value 0 infinitely often.

3. Properties of 2-representation functions. Let $A=\left\{a_{1}<a_{2}<\cdots\right\}$ be an infinite subset of $\mathbb{N}$.

3.1. Lemma. If $A$ contains $s$ elements in arithmetic progression, then $s_{2}(A) \geq s$.

Indeed, if $a, a+d, \ldots, a+(s-1) d$ all belong to $A$, then the $s$ distinct pairs $\left(b_{k}, c_{k}\right)=(a+k d, a+(s-1-k) d)$, for $0 \leq k \leq s-1$, give the $s$ representations $2 a+(s-1) d=b_{k}+c_{k}$. So $r_{2}(A, 2 a+(s-1) d) \geq s$.

3.2. Lemma. The set $2 A \backslash 2 \cdot A$ is infinite.

Indeed, for every index $i>0$, we have $a_{i}+a_{i+1} \in 2 A \backslash 2 \cdot A$, since $\frac{1}{2}\left(a_{i}+a_{i+1}\right) \notin A$, as $a_{i}<\frac{1}{2}\left(a_{i}+a_{i+1}\right)<a_{i+1}$.

3.3. LemmA. The value of $r_{2}(A, n)$ is odd if $n \in 2 \cdot A$, and it is even and positive if $n \in 2 A \backslash 2 \cdot A$. Therefore $r_{2}(A, n)$ takes infinitely often odd values and infinitely often positive even values. 
Indeed, the representations of $n$ as sum of two elements $a, b \in A$ come in pairs, $(a, b)$ and $(b, a)$, which are distinct as long as $a \neq b$. So the number $r_{2}(A, n)$ of such representations is even unless $n=2 a$ with $a \in A$, i.e. $n \in 2 \cdot A$. Moreover, both $2 \cdot A$ and $2 A \backslash 2 \cdot A$ are infinite sets, in view of the infinitude of $A$ and of 3.2 .

3.4. Proposition. If the representation function $r_{2}(A, \cdot)$ is periodically odd from some point on, then $s_{2}(A)$ is infinite.

Indeed, suppose that $r_{2}(A, n+k t)$ is odd, for given $n$ and $t$ and for each $k$ in $\mathbb{N}$. Then $n+k t \in 2 \cdot A$ for all $k \in \mathbb{N}$. All those integers $n+k t$ being even, their halves $(n+k t) / 2$ are integers forming an arithmetic progression in $A$. The result follows from 3.1.

3.5. CoROLlaRY. The representation function $r_{2}(A, \cdot)$ cannot be periodic from some point on.

Indeed, if $r_{2}(A, \cdot)$ were periodic, then it would take only a finite number of values, so that $s_{2}(A)$ would be finite. But also, $r_{2}(A, \cdot)$ would periodically take odd values, over $2 \cdot A$. So, according to $3.4, s_{2}(A)$ should be infinite, a contradiction.

3.6. THEOREM. If $s_{2}(A)$ is finite, then the representation function $r_{2}(A, \cdot)$ takes infinitely often some odd value $u$, and infinitely often some even value $v>0$. Moreover, either $r_{2}(A, \cdot)$ takes also infinitely often some other value $w \neq u, v$, or else $u \geq v+1 \geq 3$.

Proof. The representation function $r_{2}(A, \cdot)$ takes only a finite number of values, since $s_{2}(A)$ is finite. Both subsets $2 \cdot A$ and $2 A \backslash 2 \cdot A$ are infinite, according to 3.2 . On each of them, $r_{2}(A, \cdot)$ takes only a finite number of values, odd on the first subset, even on the second one, all of them not zero. Hence, it takes infinitely often an odd value $u$ on the first subset, and infinitely often an even value $v \geq 2$ on the second one.

Now suppose that $r_{2}(A, \cdot)$ does not take any other value infinitely often, that is, $r_{2}(A, \cdot)$ takes only the two values $u$ and $v$ from some point $m$ on. [Note: this implies that $A$ is an asymptotic 2-basis since $r_{2}(A, n) \neq 0$ for large enough $n$.] To show that $u \geq v+1 \geq 3$, we use power series. Let

$$
f(x)=\sum_{a \in A} x^{a}, \quad g(x)=f(x)^{2}=\sum_{n \in \mathbb{N}} r_{2}(A, n) x^{n} .
$$

Also, set

$$
\begin{aligned}
& I=\left\{n \in \mathbb{N}: r_{2}(A, n)=u, n \geq m\right\}, \\
& J=\left\{n \in \mathbb{N}: r_{2}(A, n)=v, n \geq m\right\}=[m, \infty[\backslash I .
\end{aligned}
$$

Those two sets are infinite, disjoint, and cover $[m, \infty[$. Hence, with $p(x)$ 
a polynomial, we have

$$
\begin{aligned}
g(x) & =p(x)+u \sum_{n \in I} x^{n}+v \sum_{n \in J} x^{n} \\
& =p(x)+v \sum_{n \geq m} x^{n}+(u-v) \sum_{n \in I} x^{n} \\
& =p(x)+v \frac{x^{m}}{1-x}+(u-v) \sum_{n \in I} x^{n} .
\end{aligned}
$$

The above relations are valid for $-1<x<1$, the interval of convergence on the real line of the power series $f(x)$ and $g(x)$. As $x$ approaches -1 from above, both $p(x)$ and $x^{m} /(1-x)$ converge to finite values, $p(-1)$ and $\pm 1 / 2$ respectively, while $\sum_{n \in I} x^{n}$ approaches $+\infty$ because the $n$ 's in $I$ are all even. Since $g(x)=f(x)^{2} \geq 0, u-v$ must be positive. Since $v \geq 2$, it follows that $u \geq v+1 \geq 3$.

3.7. Corollary. If $A$ is an asymptotic 2-basis, then

$$
s_{2}(A) \geq \limsup _{n \rightarrow \infty} r_{2}(A, n) \geq 3 \text {. }
$$

Indeed, if $s_{2}(A)$ is infinite, this holds trivially. If $s_{2}(A)$ is finite, then the conclusion follows from 3.6 , since $r_{2}(A, \cdot)$ cannot take the value 0 infinitely often.

3.8. Remark. As noted in the proof of 3.6, if $s_{2}(A)$ is finite and $r_{2}(A, \cdot)$ takes only two values from some point on, then $A$ is an asymptotic 2-basis. Of course no such example is available, otherwise we would know that the Erdős-Turán conjecture is not true. This raises the question: When $s_{2}(A)$ is finite, does $r_{2}(A, \cdot)$ always take infinitely often at least three distinct values?

3.9. Conclusion. Summing up, cursorily, we can say that, for a function $f: \mathbb{N} \rightarrow \mathbb{N}$ to be the 2-representation function of some infinite subset $A$ of $\mathbb{N}$, it must have at least the following properties:

(1) $f(n) \leq n+1$ for every $n \in \mathbb{N}$.

(2) $f(n)$ is even only if $n$ is odd.

(3) $f$ takes infinitely often odd values, and infinitely often positive even values.

(4) $f$ is not periodic from some point on.

(5) A fortiori, $f$ is not constant from some point on.

3.10. Application. No Sidon set can be an asymptotic 2-basis.

Indeed, $A$ is a Sidon set whenever $s_{2}(A) \leq 2$, which, in view of 3.7 , is incompatible with $A$ being an asymptotic 2-basis. 
4. Sidon sets and bases of order 3. Let $A=\left\{a_{1}<a_{2}<\cdots\right\}$ be a (finite or infinite) subset of $\mathbb{N}$. For every $n \in \mathbb{N}^{*}=\mathbb{N} \backslash\{0\}$, let

$$
\begin{aligned}
& A_{n}=\left\{a_{1}<\cdots<a_{n}\right\}, \\
& E_{n}=E_{n}(A)=\left\{a_{i}+a_{j}-a_{k}: 1 \leq i, j, k \leq n\right\}, \\
& \alpha_{n}=\min \left(\mathbb{N} \backslash 3 A_{n}\right) .
\end{aligned}
$$

All the intervals considered below are intervals in $\mathbb{N}$.

4.1. Lemma. The set $A$ is a Sidon set if and only if $a_{n+1} \in \mathbb{N} \backslash E_{n}$ for all $n$.

Indeed, first, the condition is necessary, since if $a_{n+1} \in E_{n}$ for some $n$, then, clearly, $A$ is not a Sidon set. Conversely, assume that $A$ is not a Sidon set, i.e. $a_{i}+a_{j}=a_{k}+a_{l}$ for some indices $i \leq j$ and $k \leq l$ with $(i, j) \neq(k, l)$. Then $j \neq l$ (otherwise $a_{i}=a_{k}$ and $(i, j)=(k, l)$ ), and, without loss of generality, we may assume $j<l$, i.e. $k<i$. Therefore $a_{l}=a_{i}+a_{j}-a_{k}$ with $k<i \leq j<l$, so that $a_{n+1} \in E_{n}$ for $n=l-1$.

4.2. Lemma. The set $A$ is a 3 -basis of $\mathbb{N}$ if and only if $a_{n+1} \leq \alpha_{n}$ for all $n \in \mathbb{N}^{*}$.

Indeed, since $\mathbb{N}$ is the union of all the intervals $\left[0, a_{n+1}\right.$ [ (for $n \in \mathbb{N}^{*}$ ), $A$ is a 3-basis of $\mathbb{N}$ if and only if $\left[0, a_{n+1}\left[\subset 3 A\right.\right.$ for all $n \in \mathbb{N}^{*}$. But since the sequence $\left(a_{n}\right) \subset A$ is strictly increasing, an integer $m \in\left[0, a_{n+1}\right.$ [ can only be a sum $m=a_{i}+a_{j}+a_{k}$ for $i, j, k \leq n$. So $A$ is a 3 -basis of $\mathbb{N}$ if and only if $\left[0, a_{n+1}\left[\subset 3 A_{n}\right.\right.$ for all $n \in \mathbb{N}^{*}$. Moreover, the inclusion $\left[0, a_{n+1}\left[\subset 3 A_{n}\right.\right.$, being equivalent to $\mathbb{N} \backslash 3 A_{n} \subset\left[a_{n+1}, \infty[\right.$, amounts to $a_{n+1} \leq \min \left(\mathbb{N} \backslash 3 A_{n}\right)=\alpha_{n}$.

4.3. Proposition. The set $A$ is both a Sidon set and a 3 -basis of $\mathbb{N}$ if and only if $a_{n+1} \in\left[a_{n}+1, \alpha_{n}\right] \backslash E_{n}$ for all $n \in \mathbb{N}^{*}$.

Indeed, by 4.1 and $4.2, A$ is a Sidon set and a 3 -basis of $\mathbb{N}$ if and only if for all $n \in \mathbb{N}^{*}$, we have $a_{n+1} \in \mathbb{N} \backslash E_{n}$ and $a_{n+1} \leq \alpha_{n}$. Since $a_{n+1}>a_{n}$, this amounts to $a_{n+1} \in\left(\mathbb{N} \backslash E_{n}\right) \cap\left[a_{n}+1, \alpha_{n}\right]=\left[a_{n}+1, \alpha_{n}\right] \backslash E_{n}$, for all $n \in \mathbb{N}^{*}$.

4.4. Remark. Since the properties of being a Sidon set and of being an asymptotic basis (of any order $h$ ) of $\mathbb{N}$ are translation invariant, we may always set $a_{1}=0$.

4.5. Theorem. There exists no Sidon set which is also a 3-basis of $\mathbb{N}$.

Indeed, setting $a_{1}=0$, and defining $A$ recursively by the condition $a_{n+1} \in\left[a_{n}+1, \alpha_{n}\right] \backslash E_{n}$, for every $n \in \mathbb{N}^{*}$, which characterizes Sidon sets that are 3-bases of $\mathbb{N}$ (in view of 4.3), we constructed, using the software Maple, all possible such sets $A$. It turned out that there were only finitely many such sets, which were all finite, since in every case, the sequence $\left(a_{n}\right)$ 
terminated after a few steps, due to the fact that the set $\left[a_{n}+1, \alpha_{n}\right] \backslash E_{n}$ became empty, because $\min \left(\mathbb{N} \backslash E_{n}\right)$ became more than $\alpha_{n}$.

We thus found exactly the following eleven sets:

$$
\begin{array}{ll}
\{0,1,3,7,12,20\}, & \{0,1,3,8,12,22,28,46,59\}, \\
\{0,1,3,7,12,22,30\}, & \{0,1,4,6,13,21\}, \\
\{0,1,3,8,12,18,31\}, & \{0,1,4,6,14\}, \\
\{0,1,3,8,12,18,32,45\}, & \{0,1,4,6,15,22,32\}, \\
\{0,1,3,8,12,18,34\}, & \{0,1,4,6,15,23\} . \\
\{0,1,3,8,12,22,28,45\}, &
\end{array}
$$

Since the above list exhausts all possible sets defined via the condition characterizing Sidon sets which are bases of order 3, and since each one of these sets is maximal (i.e. it cannot be enlarged any further), we conclude that there exists no Sidon set which is a 3 -basis of $\mathbb{N}$.

4.6. Lemma. Given $c \in \mathbb{N}$, let $\alpha_{n}(c)=\min \left(\left[c, \infty\left[\backslash 3 A_{n}\right)\right.\right.$. The set $A$ is a c-asymptotic 3 -basis of $\mathbb{N}$ if and only if $a_{n+1} \leq \alpha_{n}(c)$ for all $n$ such that $a_{n}>c$.

Indeed, $A$ is a $c$-asymptotic 3 -basis of $\mathbb{N}$ if and only if $[c, \infty[\subset 3 A$, i.e. for $a_{n}>c$, we have $\left[c, a_{n+1}\left[\subset 3 A_{n}\right.\right.$. The latter condition amounts to $\left[0, a_{n+1}[\subset\right.$ $3 A_{n} \cup\left[0, c\left[\right.\right.$, i.e. $\left[c, \infty\left[\backslash 3 A_{n} \subset\left[a_{n+1}, \infty\left[\right.\right.\right.\right.$, i.e. $a_{n+1} \leq \min \left(\left[c, \infty\left[\backslash 3 A_{n}\right)\right.\right.$.

4.7. Proposition. For a given $c \in \mathbb{N}$, the set $A$ is both a Sidon set and a c-asymptotic 3 -basis of $\mathbb{N}$ if and only if $a_{n+1} \in\left[a_{n}+1, \alpha_{n}(c)\right] \backslash E_{n}$ for all $n$ such that $a_{n}>c$, and $a_{n+1} \in \mathbb{N} \backslash E_{n}$ for $a_{n} \leq c$.

Indeed, by 4.1 and 4.6, $A$ is a Sidon set and a $c$-asymptotic 3 -basis of $\mathbb{N}$ if and only if $a_{n+1} \in \mathbb{N} \backslash E_{n}$ for all $n \in \mathbb{N}^{*}$ and $a_{n+1} \leq \alpha_{n}(c)$ for $a_{n}>c$. Since $a_{n+1}>a_{n}$, this amounts to $a_{n+1} \in\left(\mathbb{N} \backslash E_{n}\right) \cap\left[a_{n}+1, \alpha_{n}(c)\right]=$ $\left[a_{n}+1, \alpha_{n}(c)\right] \backslash E_{n}$ for $a_{n}>c$, while $a_{n+1} \notin E_{n}$ for all $n \in \mathbb{N}^{*}$.

4.8. Corollary. For a given $m \in \mathbb{N}^{*}$, the set $A$ is both a Sidon set and an $a_{m}$-asymptotic 3 -basis of $\mathbb{N}$ if and only if $a_{n+1} \in\left[a_{n}+1, \alpha_{n}\left(a_{m}\right)\right] \backslash E_{n}$ for all integers $n>m$, and $a_{n+1} \in \mathbb{N} \backslash E_{n}$ for $1 \leq n \leq m$.

4.9. Remark. In view of 4.4, we may and do assume that $\mathbf{a}_{\mathbf{1}}=\mathbf{0}$ in the following. Then $A$ is a Sidon set and an $a_{1}$-asymptotic 3-basis of $\mathbb{N}$ if and only if $A$ is a Sidon set and a 3 -basis of $\mathbb{N}$, which is impossible in view of 4.5. Thus, if $A$ is to be a Sidon set and an asymptotic 3-basis of $\mathbb{N}$, then it must be a Sidon set and an $a_{m}$-asymptotic 3 -basis of $\mathbb{N}$ for some $m \geq 2$. We show next that $m \geq 3$, and later indicate that in fact $m \geq 4$. We further report on the results of some computations establishing a lower bound for $c$ such that $A$ is a $c$-asymptotic 3 -basis of $\mathbb{N}$ (if such a basis exists). 
4.10. Application. There is no Sidon set which is an $a_{2}$-asymptotic 3-basis of $\mathbb{N}$.

Indeed, assume that $\left[a_{2}, \infty\left[\subset 3 A\right.\right.$. Then $a_{2} \geq 2$; otherwise, $A$ would be a 3 -basis of $\mathbb{N}$ and a Sidon set, which contradicts 4.5. Also, since $a_{2}+1 \in 3 A$, we must have $a_{3}=a_{2}+1$; for, as $a_{2}<a_{2}+1<2 a_{2}$, the only way to have $a_{2}+1=a_{i}+a_{j}+a_{k}$, with necessarily $1 \leq i, j, k \leq 3$, is to have $i=j=1$ and $k=3$. Moreover, since $a_{2}+2 \in 3 A$, it follows that $a_{2}=2$; otherwise, $a_{2} \geq 3$ implies $a_{3}<a_{2}+2<2 a_{2}$, and since a Sidon set cannot contain three terms in arithmetic progression, $a_{4}-a_{3} \neq a_{3}-a_{2}=1$, i.e. $a_{4}>a_{3}+1=a_{2}+2$, which makes $a_{2}+2=a_{i}+a_{j}+a_{k}$ impossible. Thus, starting with $a_{1}=0, a_{2}=2, a_{3}=3$, and defining $A$ recursively by the condition $a_{n+1} \in\left[a_{n}+1, \alpha_{n}\left(a_{2}\right)\right] \backslash E_{n}$ for all $n \geq 3$, which characterizes Sidon sets that are $a_{2}$-asymptotic 3 -bases of $\mathbb{N}$ (in view of 4.8 ), we constructed all possible such sets $A$. All we found were the following eleven finite maximal such sets:

$$
\begin{array}{ll}
\{0,2,3,7,13,21,30\}, & \{0,2,3,8,12,19,34,47\}, \\
\{0,2,3,7,13,22,30\}, & \{0,2,3,8,12,19,37\}, \\
\{0,2,3,7,15,21,31\}, & \{0,2,3,8,15,19\}, \\
\{0,2,3,7,15,21,32\}, & \{0,2,3,9\}, \\
\{0,2,3,8,12,19,32\}, & \{0,2,3,10\} . \\
\{0,2,3,8,12,19,33\}, &
\end{array}
$$

Since this list is exhaustive, we conclude that there is no Sidon set which is an $a_{2}$-asymptotic 3 -basis of $\mathbb{N}$.

4.11. Computation Results. If a Sidon set $A$ is also a $c$-asymptotic 3-basis of $\mathbb{N}$, then $c \geq 40$.

Indeed, for a given positive integer $c \leq 39$, there are only finitely many Sidon sets contained in $[0, c]$, which are easy to enumerate by setting $a_{1}=0$ and applying the criterion in 4.1. Then, starting from each one of these Sidon sets and applying the algorithm in 4.7, we computed, using the software Maple, all possible such maximal Sidon sets: they were all finite and there were only finitely many of them for each $c \leq 39$.

Thus, for instance, for $c=39$, we found 211795 maximal sets each of cardinality $\leq 23$. They obviously include all maximal sets corresponding to smaller values of $c$. An example of a maximal set of maximal length for $c=39$ is

$$
\{0,3,9,11,25,37,38,42,57,78,102,125,168,175,
$$


Also, by similar arguments to those in 4.10 , and by more extensive computations, we found that there is no Sidon set which is an $a_{3}$-asymptotic 3-basis of $\mathbb{N}$.

\section{References}

[1] P. Erdős, A. Sárközy and V. T. Sós, On additive properties of general sequences, Discrete Math. 136 (1994), 75-99.

[2] P. Erdős and P. Turán, On a problem of Sidon in additive number theory, and on some related problems, J. London Math. Soc. 16 (1941), 212-215.

[3] A. Sárközy, Unsolved problems in number theory, Period. Math. Hungar. 42 (2001), 17-35.

Université Jean Monnet

23 rue Michelon

42023 St-Etienne, France

E-mail: grekos@univ-st-etienne.fr

Penn State University

25 Yearsley Mill Rd.

Media, PA 19063, U.S.A.

E-mail: cxh22@psu.edu
120 rue de Charonne

75011 Paris, France E-mail: labib.haddad@wanadoo.fr

Math.-Stat. Department P.O. Box 68 (G. Hällströmin katu 2b) FIN-00014 University of Helsinki, Finland E-mail: pihko@users.csc.fi

Received on 11.9.2006

and in revised form on 28.6.2007 